\title{
AVALIAÇÃO DA TÉCNICA FOTOGRÁFICA NAS AUTOPSIAS FORENSES DO INSTITUTO MÉDICO LEGAL DE PORTO VELHO - RO, NO BRASIL
}

EVALUATION OF THE PHOTOGRAPHIC TECHNIQUE IN THE FORENSIC AUTOPSIAS OF THE INSTITUTE

MEDICO LEGAL DE PORTO VELHO - RO, BRAZIL

\author{
Os autores informam não haver conflito de interesse
}

ARTIGO ORIGINAL recebido 25/03/2021, aceito em 04/06/2021

Como citar: Valente M, Sobral LLG, Queiroga Jr G. Avaliação da técnica fotográfica nas autópsias forenses do Instituto Médico Legal de Porto Velho - RO, no Brasil. Persp Med Legal Pericias Med. 2021; 6: e210607

https://dx.doi.org/10.47005/210607

(1) Departamento de Saúde Pública e Ciências Forenses e Educação Médica da Faculdade de Medicina da Universidade do Porto, Porto, Portugal (autor principal)

(2) Instituto Médico Legal de Porto Velho, Polícia Civil do Estado de Rondônia, Brasil (autor principal)

e-mail: medicolegista-murilo@valente-aguiar.med.com.br

\section{RESUMO}

A fotografia nas autópsias forenses de morte violenta tem a finalidade de documentar as provas materiais do delito e orientar as decisões nos tribunais de justiça. Neste trabalho testamos a hipótese de que as fotografias atuais, apresentadas nos relatórios forenses do Instituto Médico Legal Dr. José Adelino da Silva, em Porto Velho, localizado na capital do Estado de Rondônia, no Brasil, seriam inadequadas por não cumprirem os critérios que mais contribuem para a qualidade final da fotografia forense. Através de uma análise retrospectiva, avaliamos a qualidade das fotografias incorporadas nos relatórios das autópsias forenses relativos a morte violenta. Concluímos que mais da metade das fotografias incorporadas $(52,6 \%)$ eram inviáveis do ponto de vista técnico e, no que concerne esse critério, não deveriam ter sido utilizadas nos relatórios. Considerando as limitações encontradas, sugere-se estratégias a serem adotadas no Instituto para as corrigir, sendo primordial a elaboração de um protocolo com os procedimentos harmonizados para o registro fotográfico das autópsias forenses tendo em vista a melhoria dos relatórios periciais forenses.

Palavras-chave: Fotografia forense, Autópsia forense, Fotografia especializada, Fotografia digital. 


\section{ABSTRACT}

Photography in forensic autopsies of violent death is intended to document the material evidence of the offense and guide decisions in the courts of law. In this paper, we tested the hypothesis that the current photographs, presented in the forensic reports of the Dr. José Adelino da Silva Legal Medical Institute, in Porto Velho, located in the capital of the State of Rondônia, in Brazil, would be inappropriate for not meeting the criteria that must contribute to the final quality of forensic photography. Through a retrospective analysis, we evaluated the quality of the photographs incorporated in the reports of the forensic autopsies related to violent death. We concluded that more than half of the incorporated photographs (52.6\%) were not technically feasible and, regarding this criterion, they should not have been used in the reports. Considering the limitations found, it is suggested strategies to be adopted at the Institute to correct them, being essential the elaboration of a protocol with the harmonized procedures for the photographic record of the forensic autopsies with a view to improving the forensic expert reports.

Keywords: Forensic photography, Forensic autopsy, Specialized photography, Digital photography.

\section{INTRODUÇÃO}

A documentação fotográfica constitui um dos elementos materiais, de natureza objetiva, da prova do crime. É um importante meio de coleta e preservação das evidências físicas e que contribuir para o julgamento correto dos processos nos tribunais de justiça (1). Para tanto, é necessário que, esses registros fotográficos, tenham qualidade suficiente para que possa transmitir aquilo que foi de fato observado (1-3).

O Código de Processo Penal Brasileiro (CPP) criado através do Decreto-Lei no 3.689 de 3 de outubro de 1941, nos seus Artigos no 164 e 165, orienta para que os peritos utilizem a fotografia como uma das provas do delito a serem juntadas ao laudo dentre as imagens utilizadas. Porém, o CPP não determina como essas fotografias devam ser elaboradas.

Na publicação do Decreto-Lei que criava o CPP ainda não era patente a utilização da fotografia digital sendo as fotografias capturadas em filmes analógicos e, após revelação e impressão, eram encaminhadas junto ao relatório pericial. No final da década de 80, o método analógico começou a ser substituído pelo digital, onde a facilidade do processamento apresentava múltiplas vantagens, e as fotografias passaram a ser impressas diretamente nos relatórios e não mais anexadas aos mesmos. Esta facilidade da incorporação das fotografias diretamente nos relatórios entra em conflito com a necessidade de conhecimentos fotográficos específicos e de treinamento dos peritos para a utilização dos programas de computadores que processam e ajustam as imagens que vão ser incorporadas. A alienação destes conhecimentos resulta em fotografias de má qualidade que pouco ou nada auxiliam nos julgamentos dos operadores do direito.

No Instituto Médico Legal Dr. José Adelino da Silva, em Porto Velho (IML/PVH/RO), localizado na capital do Estado de Rondônia, no Brasil, não existe um Procedimento Operacional Padrão (POP) de Fotografia Forense, bem como não existe, em seu quadro funcional, a figura do fotógrafo forense. Essas inexistências autorizam os médicos e os odontólogos do Instituto a capturarem ou não as fotografias durante suas perícias e, se capturadas, que essas sejam incorporadas ou não aos seus relatórios.

Neste trabalho, partimos do pressuposto de que as fotografias incorporadas nos relatórios forenses do Instituto são inadequadas do ponto de vista técnico e, se confirmado, sugeriríamos as estratégias para correção das vulnerabilidades.

\section{METODOLOGIA}

Foi realizado um estudo retrospectivo baseado na análise de todos os relatórios de autópsia forense, elaborados no IML/PVH/RO, entre 01 de janeiro 2016 e 31 de dezembro de 2017 tendo, como critério de inclusão, os relativos à morte violenta e que incorporassem fotografias.

As fotografias incorporadas nos relatórios foram 
analisadas baseando-se em 9 critérios considerados como aqueles que mais contribuem para a qualidade final da fotografia forense e, consequentemente, sua fiabilidade como prova pericial:

1) Nitidez da fotografia;

2) Foco da fotografia;

3) Cor da fotografia;

4) Exposição da fotografia;

5) Inclusão do número do processo na fotografia;

6) Inclusão de testemunho métrico na fotografia;

7) Plano de fundo da fotografia;

8) Inclusão de referências anatômicas no enquadramento da fotografia;

9) Tamanho da fotografia.

Cada uma destas variáveis foi avaliada de acordo com a escala usada pelo Instituto Nacional de Medicina Legal e Ciências Forenses - Delegacia do Norte em Porto (4), Portugal, na realização de auditorias, modificada e adaptada para este estudo, onde foram utilizados 5 graus de qualidade $(1,2,3$, 4, e 5) com o seguinte significado:

a) 1 (inadequada - fotografia elaborada incorretamente);

b) 2 (parcialmente adequada - fotografia com deficiências importantes que inviabilizam a sua utilização nos relatórios);

c) 3 (adequada - fotografia mal elaborada, porém as deficiências não inviabilizam a sua incorporação nos relatórios);

d) 4 (relevante - fotografia elaborada adequadamente, com ligeiras deficiências, a corrigir);

e) 5 (muito boa - fotografia elaborada corretamente).

Para garantir a fiabilidade e a uniformidade, nos critérios de qualidade das fotografias forenses incorporadas aos relatórios, todos os dados foram colhidos pelo autor, bem como a análise e a classificação das fotografias.

\section{RESULTADOS}

No período em estudo (01 de janeiro de 2016 a 31 de dezembro de 2017), foram realizadas 920 autópsias forenses por morte violenta no IML/PVH/RO, das quais, apenas 99 (10,8\%) continham fotografias incorporadas nos relatórios (Fig. 1 e Fig. 2).

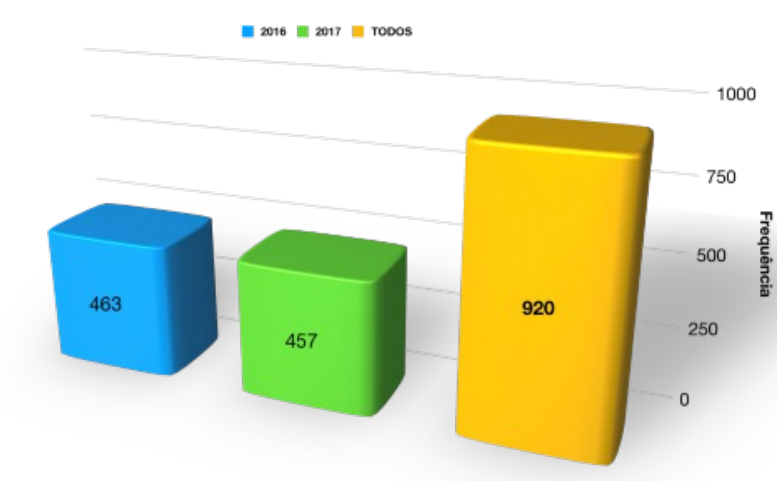

Fig. 1-Quantidade de autópsias forenses por morte violenta realizadas no IML/PVH/RO no período de 01 JAN 2016 a 31 DEZ 2017.

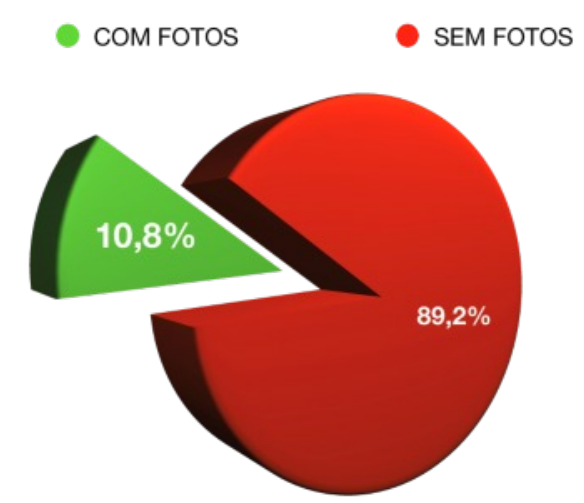

Fig. 2 - Porcentagem dos laudos com e sem fotografias incorporadas nas autópsias forenses por morte violenta realizadas no IML/PVH/RO no período de 01 JAN 2016 a 31 DEZ 2017.

$\mathrm{O} \mathrm{IML} / \mathrm{PVH} / \mathrm{RO}$ tem, em seu quadro funcional, 27 médicos legistas e 3 odontólogos legais atuando regularmente e não possui um fotógrafo forense. Todas as fotografias foram executadas pelos peritos, responsáveis pela autópsia, utilizando suas câmeras fotográficas particulares ou seus smartphones. No período do estudo, 9 dos médicos e 2 dos odontólogos incorporaram fotografias em seus relatórios.

Foram analisadas, individualmente, 481 fotografias que receberam um escore para cada um dos critérios de qualidade. As somatórias dos escores classificaram 39 fotografias $(8,1 \%)$ como INADEQUADAS, 214 (44,5\%) como PARCIALMENTE ADEQUADAS, 175 (36,4\%) como ADEQUADAS e 53 $(11,0 \%)$ como RELEVANTES (Fig. 3). Não foram encontradas fotografias na categoria MUITO BOA. 


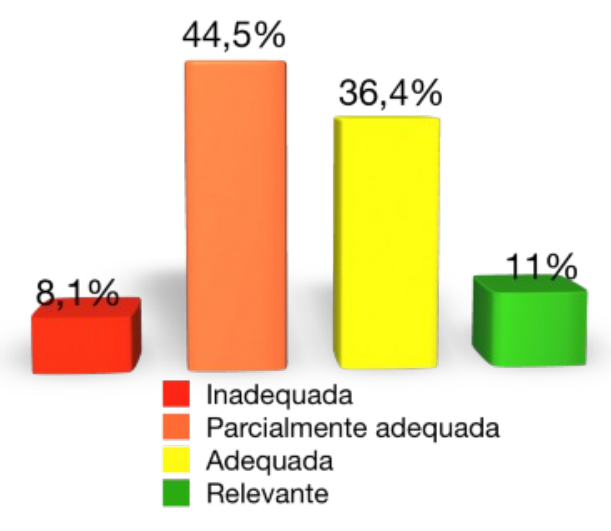

Fig. 3 - Classificação final das fotografias.

O escore médio dos critérios analisados, em cada foto, classificou 253 fotografias (52,6\%) como inviáveis para incorporação nos relatórios e 228 $(47,4 \%)$ classificadas como viáveis para incorporação nos relatórios (Fig. 4).

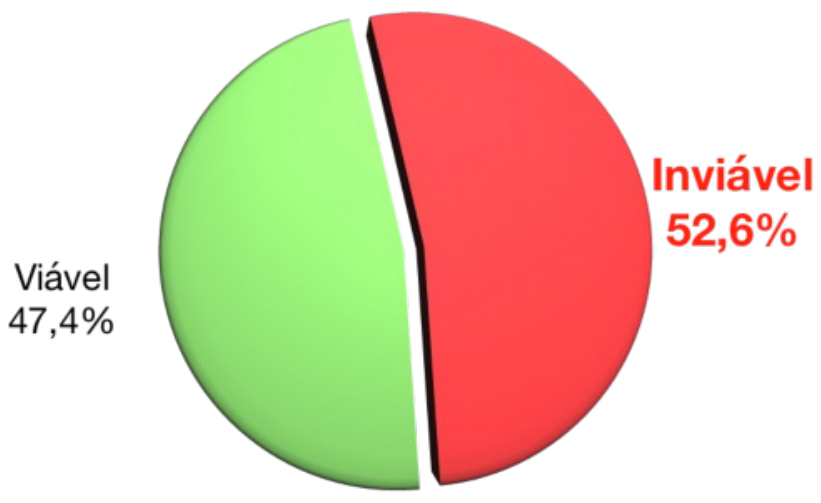

Fig. 4 - Viabilidade das fotografias incorporadas nas autópsias forenses por morte violenta realizadas no IML/PVH/RO no período de 01 JAN 2016 a 31 DEZ 2017.

Os quatro critérios que inviabilizaram a incorporação das fotografias nos relatórios foram: COR DAS FOTOGRAFIAS, INCLUSÃO DO № DO PROCESSO, INCLUSÃO DO TESTEMUNHO MÉTRICO e o TAMANHO DAS FOTOGRAFIAS (Fig. 5).

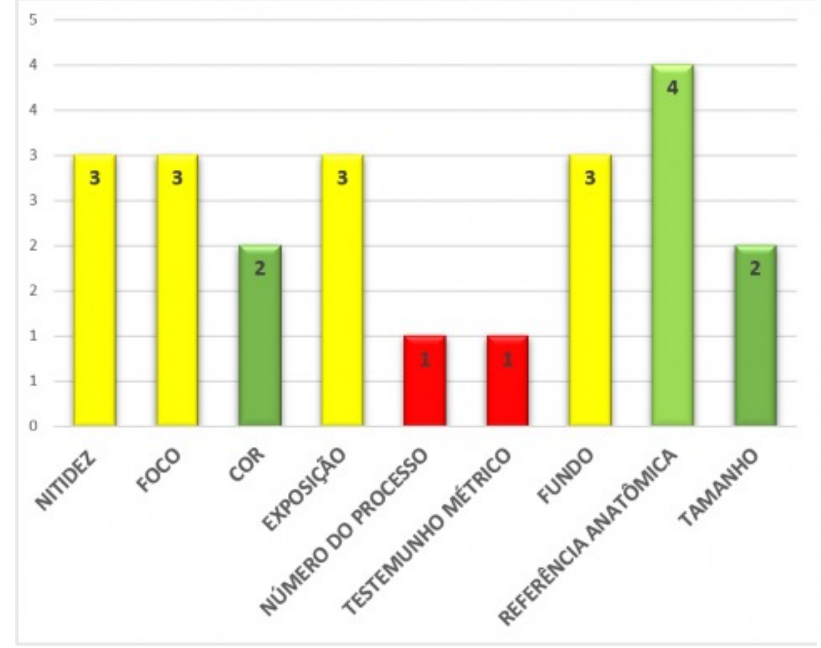

Fig. 5 - Média dos escores dos critérios de avaliação das fotografias incorporadas nas autópsias forenses por morte violenta realizadas no IML/PVH/RO no período de 01 JAN 2016 a 31 DEZ 2017.

A análise individual dos critérios apresentou os seguintes resultados (Tabela 1):

\begin{tabular}{|c|c|c|c|c|c|}
\hline \multirow{2}{*}{$\begin{array}{l}\text { CRITÉRIO } \\
\text { AVALIADO }\end{array}$} & \multicolumn{5}{|c|}{ CLASSIFICAÇÃOO } \\
\hline & INADEQUADA & $\begin{array}{l}\text { PARCIALMENTE } \\
\text { ADEQUADA }\end{array}$ & ADEquADA & RELEVANTE & мUाто вом \\
\hline NITIDEZ DA & 26 & 44 & 149 & 132 & 130 \\
\hline FOTOGRAFIA & $(5,4 \%)$ & $(9,126)$ & $(31,066)$ & $(27,4 \%)$ & $(27,006)$ \\
\hline FOCO DA & 39 & 56 & 144 & 132 & 110 \\
\hline FOTOGRAFIA & $(8,1 \%)$ & $(11,6 \% 6)$ & $(29,9 \% 6)$ & $(27,496)$ & $(22,99 \%)$ \\
\hline SORRA ГRTOCAF & 368 & 13 & 18 & 32 & 50 \\
\hline COK DA FOIOGAFIA & {$[76,5 * 6)$} & $(2,7 \%)$ & $(3,7 \%)$ & $(6,7 \% 6)$ & $(10,4 \%)$ \\
\hline EXPOSIÇĊO DA & 59 & 123 & 181 & 70 & 48 \\
\hline FOTOGRAFIA & {$[12,36)$} & $(25,6 \%)$ & $(37,6 \% 6)$ & $(14,69)$ & $(10,0 \%)$ \\
\hline INCLUSÃO DO & 481 & 0 & 0 & 0 & 0 \\
\hline PROCESSO & $(100,050)$ & $(0,065)$ & $(0,0 \% 5)$ & $(0,050)$ & {$[0,050)$} \\
\hline TESTEMUNHO & 481 & 0 & 0 & 0 & 0 \\
\hline MÉtraco & $(100,066)$ & $(0,0 \%)$ & $(0,00 \%)$ & $(0,0 \%)$ & $(0,0 \%)$ \\
\hline FUNDO DA & 20 & 60 & 174 & 103 & 124 \\
\hline FOTOGRAFIA & $(4,280)$ & $(12,596)$ & $(36,2 \times 6)$ & $(21,4 \%)$ & $(25,8 \%)$ \\
\hline REFERĖNCIA & 97 & 42 & 34 & 52 & 257 \\
\hline ANATOOMICA & $(20,266)$ & $(8,796)$ & $(7,1 \%)$ & $(10,866)$ & $(53,446)$ \\
\hline TAMANHO DA & 173 & 157 & 39 & 59 & 53 \\
\hline FOTOGRAFIA & $(36,066)$ & $(32,646)$ & $(8,1 \%)$ & (12,36) & (11,06) \\
\hline
\end{tabular}

TABELA 1 - CROSSTAB - Classificação dos critérios de qualidade avaliados 


\section{DISCUSSÃO}

A evolução da tecnologia dos sensores dos equipamentos eletrônicos, que captam e processam imagens, associada à facilidade do manuseio do equipamento, onde não se faz necessário a habilitação técnica para processar o rolo de filme com produtos químicos tóxicos (5), tornou obsoleta a imagem do fotógrafo clássico, pois qualquer pessoa consegue fotografar e disponibilizar suas imagens instantaneamente. Porém, quando a qualidade da arte fotográfica é fundamental, como ocorre nas perícias forenses, e as fotografias serão usadas como provas materiais do delito que orientarão as decisões dos operadores de direito, se faz necessário o implemento dos critérios de qualidade nos registros fotográficos.

Nos relatórios de autópsias forenses, especificamente aos relativos à morte violenta, além dos conhecimentos de anatomia humana, se faz necessário o conhecimento básico na área da fotografia bem como um equipamento fotográfico de boa qualidade, uma impressora colorida com qualidade fotográfica e escalas métricas ABFO no2. Os conhecimentos dos peritos em anatomia humana colaboraram com o resultado da melhor classificação obtida das fotografias analisadas, justamente no critério da INCLUSÃO DE REFERÊNCIA ANATÔMICA na fotografia, onde 53,4\% foram classificadas no grau de qualidade 5 - MUITO BOA (Tabela 1 - Destaque em verde) e isso é justificado pelo fato de serem os médicos e odontólogos os responsáveis pelas capturas das imagens. A não inclusão de referências anatômicas pode criar interpretações errôneas da localização das lesões e inviabilizar a incorporação da fotografia no relatório da autópsia.

Os critérios considerados fundamentais na fotografia forense, dentro dos analisados, são: a NITIDEZ, o FOCO, a EXPOSIÇÃO e a COR das fotografias. Todos esses critérios são diretamente dependentes da qualidade do equipamento fotográfico, do conhecimento do perito na área da fotografia e no domínio do equipamento utilizado. A maioria das fotografias nos critérios da NITIDEZ, do FOCO e da EXPOSIÇÃO foram classificadas apenas como ADEQUADAS $(31,0 \%, 29,9 \%$ e $37,6 \%$ respectivamente) e isso é justificado mais pela qualidade dos equipamentos utilizados do que pelo conhecimento na área da fotografia e/ou domínio do equipamento fotográfico. Pois o curso de fotografia forense, ministrado durante a formação de Perito Oficial na Academia de Polícia Civil, está voltado, somente, para a fotografia do local de crime (Tabelas 1 - Destaque em azul). Já o critério da COR da fotografia, além da qualidade do equipamento, necessita de uma impressora com qualidade fotográfica e devidamente calibrada. $\mathrm{O}$ IML/PVH/RO não possui uma impressora colorida e este fato justifica o resultado da classificação das fotografias neste critério de qualidade como INADEQUADAS (76,5\%) (Tabela 3). E, neste critério, apenas 100 (20,8\%), das 481 fotografias analisadas, tinham condições de serem incluídas nos relatórios das autópsias realizadas. A ausência de uma impressora colorida é a principal justificativa dos peritos de não incluírem as imagens capturadas nas suas perícias. As fotografias coloridas incorporadas nos relatórios analisados foram produzidas nos equipamentos de impressão particulares dos peritos, muitas das vezes, de qualidade inferior à necessária.

A inclusão, nos critérios de qualidade, do PLANO DE FUNDO das fotografias, visava a avaliação da capacidade do perito de destacar o que era relevante a ser mostrado aos operadores do direito, eliminando os elementos na fotografia que, de alguma forma, pudessem distrair a atenção e interpretação fiel do achado. Neste quesito, a maioria das fotografias analisadas foram classificadas como ADEQUADAS (36,2\%) (Tabela 1 - Destaque em azul).

Outro critério que inviabilizou a incorporação das fotografias nos relatórios foi o critério do TAMANHO da fotografia. Imagens muito pequenas não permitem a definição precisa da prova material do delito e não esclarecem as dúvidas dos operadores do direito. Isso faz com que, muitas das vezes, a autoridade judiciária solicite ao perito, e não ao Instituto, as imagens originais para poder orientar as decisões nos tribunais de justiça, já que as imagens ficam de posse do perito e não do Instituto. O IML/PVH/RO não dispõe de estação de trabalho, incluída em um servidor criptografado, onde todas as fotografias capturadas nas perícias fiquem arquivadas e protegidas pela cadeia de custódia. Logo, $330(68,6 \%)$ das 481 fotografias incorporadas foram classificadas como INADEQUADAS $(36,0 \%)$ e PARCIALMENTE ADEQUADAS $(32,6 \%)$ e, portanto, 
inviáveis para serem utilizadas nos relatórios (Tabela 1 - Destaque em vermelho).

Os piores resultados foram obtidos nos critérios da INCLUSÃO DO NÚMERO DO PROCESSO e da INCLUSÃO DE TESTEMUNHO MÉTRICO, ambos classificados como INADEQUADOS nas 481 (100\%) fotografias incorporadas nos relatórios (Tabelas 1 - Destaque em vermelho). Esses dois critérios são questões basilares e imprescindíveis. A inclusão do número do processo é a garantia de que determinada fotografia pertence ao caso periciado e afaste qualquer dúvida de que possa pertencer a outra perícia. Já a inclusão do testemunho métrico assegura uma noção de escala independentemente do tamanho da ampliação utilizada na fotografia. No âmbito jurídico, uma fotografia corretamente elaborada tem o poder de orientar as decisões nos tribunais da justiça por eliminar a subjetividade da descrição. A fiel documentação fotográfica do caso garante que os operadores do direito possam julgar com segurança. No âmbito forense, ajuda o perito a descrever corretamente os achados em seus relatórios, realçando aspectos difíceis e que, por vezes, passaram despercebidos ou não foram observados durante a autópsia; permite o compartilhamento das informações para discussão entre os seus pares e podem ser a matéria prima a ser agregada na formação e atualização de profissionais.

\section{CONCLUSÃO}

A carência de normalização, no registro fotográfico das autópsias forenses no IML/PVH/RO, permitiu que os médicos e os odontólogos tivessem a liberdade de capturar e incorporar as imagens nos relatórios sem os requisitos de qualidade necessários. Em função disso, foram obtidas fotografias de má qualidade e de pouco uso na matéria criminal, onde mais da metade das fotografias $(52,6 \%)$, foram inviáveis e não poderiam ter sido incorporadas nos relatórios das autópsias analisados (Fig. 4).

As falhas encontradas, nos critérios de qualidade avaliados, podem ser corrigidas com a adoção das seguintes estratégias:

a) Elaboração de um POP com os procedimentos harmonizados para os registros fotográficos nas autópsias forenses, de forma prática e economicamente viável, que apresente um produto final de qualidade e que garanta a fiabilidade da prova material;

b) Criação de uma equipe para implementação de auditorias de fotografia forense, com caráter regular, para monitorizar o cumprimento do protocolo com os procedimentos harmonizados para os registros fotográficos das autópsias forenses;

c) Realização regular e contínua de cursos de fotografia forense voltados a valorização de competências em fotografia forense para todos os funcionários que participam do exame pericial no IML/PVH/RO;

d) Adequação do curso de fotografia forense na Academia de Polícia Civil do Estado de Rondônia, priorizando os conhecimentos voltados para a documentação correta das autópsias forenses nos cursos de formação de peritos oficiais, médicos e odontólogos, bem como na formação dos técnicos de necrópsia;

e) Aquisição de duas câmeras fotográficas digitais de boa qualidade, permitindo o trabalho de duas equipes simultaneamente, e uma impressora com qualidade fotográfica para o IML/PVH/RO;

f) Aquisição de escalas ABFO \#2;

g) Dispor uma estação de trabalho, em uma rede segura, para a criação de um banco de imagens com o arquivamento de todas as fotografias realizadas no IML/PVH/RO, permitindo a indexação das fotografias aos devidos processos e garantindo a cadeia de custódia das imagens.

\section{BIBLIOGRAFIA}

1. Rost $\mathrm{T}$, Wittig $\mathrm{H}$. [Photodocumentation during autopsy]. Archiv fur Kriminologie. 2014;233(1-2):5766.

2. Henham AP, Lee KA. Photography in forensic medicine. The Journal of audiovisual media in medicine. 1994;17(1):15-20.

3. Aguiar MSV. Manual Prático de Fotografia Forense - com casos comentados. 1a ed. São Paulo: Fontenele Publicações; 2020. 164 p.

4. Marques A, Santos A, Vieira DN. Norma procedimental : Recomendações Gerais Sobre Fotografia Forense. NP - INMLCF - 010. 2013.

5. Redsicker DR, Gordner G, James SH, Laws AC, Redsicker AD. The Practical Methodology of Forensic Photography. 2nd Ed. ed. Boca Raton, NY: CRC Press LLC; 2001. 